1 Different strategies to initiate and maintain hyperventilation: their effect on

2 continuous estimates of dynamic cerebral autoregulation

3 Jatinder S. Minhas ${ }^{1}$, Charlotte Kennedy ${ }^{1}$, Thompson G Robinson ${ }^{1,2}$,

4 Ronney B Panerai ${ }^{1,2}$

$5{ }^{1}$ Cerebral Haemodynamics in Ageing and Stroke Medicine (CHIASM) Cardiovascular 6 Sciences Research Group, Department of Cardiovascular Sciences, University of Leicester, 7 Leicester, United Kingdom.

$8 \quad{ }^{2}$ National Institute for Health Research Leicester Biomedical Research Centre, Leicester, 9 United Kingdom

Correspondence to Jatinder S. Minhas, University of Leicester, Department of Cardiovascular

Sciences, Leicester, LE2 7LX, United Kingdom,+44 116252 5841, Email: jm591@1e.ac.uk

ORCID ID JS Minhas: 0000-0002-0576-9105

Running Title: Strategies to initiate and maintain hyperventilation and dCA

Key words: Respiration, Carbon Dioxide, Haemodynamics, Hypocapnia, Hyperventilation, Acute Stroke 


\section{Abbreviations list}

18

19

20

ARI Autoregulation index

21 BP

Blood pressure

22 CA

Cerebral autoregulation

$23 \mathrm{CBF}$

Cerebral blood flow

24 CBFV

Cerebral blood flow velocity

$25 \mathrm{CO}_{2}$

Carbon dioxide

$26 \mathrm{CrCP}$

Critical closing pressure

27 CVRi

Cerebrovascular resistance index

$28 \mathrm{dCA}$

Dynamic cerebral autoregulation

29 ECG

Electrocardiogram

$30 \quad \mathrm{EtCO}_{2}$

End-tidal $\mathrm{CO}_{2}$

31 HR

Heart rate

32 MABP

Mean arterial blood pressure

33 MCA

Middle cerebral artery

$34 \mathrm{PaCO}_{2}$

Partial pressure carbon dioxide

35 RAP

Resistance-area product

36 SD

Standard deviation

37 TCD

Transcranial Doppler 
Abstract

\section{Objective}

Capnography is a key monitoring intervention in several neurologically vulnerable clinical states. Cerebral autoregulation (CA) describes the ability of the cerebrovascular system to maintain a near constant cerebral blood flow (CBF) throughout fluctuations in systemic arterial blood pressure (ABP), with the partial pressure of arterial carbon dioxide $\left(\mathrm{PaCO}_{2}\right)$ known to directly influence CA. Previous work has demonstrated dysautoregulation lasting around 30 seconds prior to the anticipated augmentation of hyperventilation-associated hypocapnia. In order assess to potential benefit of hypocapnic interventions in an acute stroke setting, minimisation of dysregulation is paramount.

\section{Approach}

Hyperventilation strategies to induce and maintain hypocapnia were performed in 61 healthy participants, effects on temporal estimates of dCA (autoregulation index, ARI) were assessed to validate the most effective strategy for inducing and maintaining hypocapnia.

\section{Main Results}

The extent of initial decrease was significantly smaller in the continuous metronome strategy compared to the delayed metronome and voluntary strategies ( $\triangle \mathrm{ARI} 0.33 \pm 1.18,2.80 \pm 3.33$ and $3.69 \pm 2.79$ respectively, $\mathrm{p}<0.017)$.

\section{Significance}

The use of a continuous metronome to induce hypocapnia rather than the sudden inception of an auditory stimulus appears to reduce the initial decrease in autoregulatory capacity seen in previous studies. Dysautoregulation can be minimised by continuous metronome use during hyperventilation-induced hypocapnia. This advancement in understanding of the behaviour of CA during hypocapnia permits safer delivery of CA targeted interventions, particularly in neurologically vulnerable patient populations. 
Cerebral autoregulation (CA) describes the ability of the cerebrovascular system to maintain a near constant cerebral blood flow (CBF) throughout fluctuations in systemic arterial blood pressure (ABP) (Lassen 1959). CA is impaired in a multitude of acute neurological conditions (Patel et al 2016, Minhas et al 2018). Between 60-150mmHg CBF tends to be independent of changes in ABP due to vessel diameter modifications, which alter the cerebrovascular resistance (Lassen 1959, Paulson et al 1990). CA can be described in terms of its static or dynamic properties. Static CA represents the steady-state relationship between ABP and CBF and is used to describe the response of the cerebrovascular system to sustained changes in BP. Dynamic CA (dCA) describes the regulation of CBF in response to acute BP perturbations and therefore evaluates the temporal aspects of CA as well as the degree of functioning (Tiecks et al 1995, Panerai 2008).

A key vasoactive stimulus often used in CA studies is the elevated partial pressure of arterial carbon dioxide $\left(\mathrm{PaCO}_{2}\right)$, which is known to directly influence CA (Aaslid et al 1989, Birch et al 1995, Panerai et al 1999, Ainslie et al 2008, Ogoh et al 2008, Dineen et al 2010). Specifically, hypercapnia impairs dCA and hypocapnia augments dCA, as demonstrated in a number of previous studies (Aaslid et al 1989, Birch et al 1995, Panerai et al 1999, Dineen et al 2010, Ogoh et al 2010). Continuous recordings of end-tidal $\mathrm{CO}_{2}\left(\mathrm{EtCO}_{2}\right)$ are increasingly used in physiological and clinical studies as surrogate estimates of $\mathrm{PaCO}_{2}$. Capnographic estimates of $\mathrm{PaCO}_{2}$ have been shown to be useful for continuously monitoring the respiratory status of patients in intensive care settings (Rittayamai et al 2014, Long et al 2017, Minhas 2018). Furthermore, international guidelines advocate the use of capnography in acute stroke patients undergoing thrombectomy, demonstrating further opportunities to evolve our understanding of $\mathrm{PaCO}_{2}$ change during this neurologically vulnerable period (Royal College of Physicians Intercollegiate Stroke Working Party, 2016). In addition to applications in exercise physiology and respiratory diseases, studies of the cerebral circulation require assessment of $\mathrm{PaCO}_{2}$ changes due to its potent effects on CBF (Battisti-Charbonney et al 2011). Importantly, $\mathrm{PaCO}_{2}$ changes can be induced using respiratory manoeuvres; the variance in $\mathrm{EtCO}_{2}$ concentration measured being assumed analogous to that of the arterial concentration. Hypercapnia can be induced using several methods including rebreathing techniques, breath holding and direct inhalation of $\mathrm{CO}_{2}$ (Markus and Harrison 1992). Conversely, the only method of obtaining hypocapnia in humans is to induce hyperventilation, either mechanically or voluntarily, dependent on the subject population (Newell et al 1996, Ide et al 2003, Eames et 
al 2004, Ogoh et al 2008, Ogoh et al 2010, Minhas et al 2018a). It is therefore pertinent to refine the methods used to induce and maintain hyperventilation in order to obtain the most valid and reproducible results.

Hypocapnia has previously been used in the management of acute brain injury to reduce intracranial pressure (Takahashi et al 2014). Novel interventional studies are underway designed to target CA augmentation via hypocapnia in an acute stroke setting, in an effort to harbour preferential clinical effects including lower ICP and penumbral protection in the presence of further ischemic insults (Minhas et al 2018a). Hypocapnia has been used as a neuroprotective mechanism to improve CA in subarachnoid haemorrhage (Ma et al 2000), patients with liver failure (Strauss 2007) and during anesthesia with isoflurane (McCulloch et al 2005). These studies have provided a key translational platform for the BREATHE-ICH study (ClinicalTrials.gov identifier NCT03324321) demonstrating a direct application for the improved understanding of CA manipulation using hypocapnia (Minhas et al 2018a).

A novel analysis approach by Dineen et al (2010) used a moving-window autoregressive moving average (MW-ARMA) model to explore the temporal patterns of dCA during respiratory manoeuvres. On evaluation, an initial decrease in autoregulation was demonstrated during hyperventilation-associated hypocapnia. This dysautoregulation lasted around 30 seconds prior to the anticipated augmentation (Dineen et al 2010). Previously used batchprocessing analysis methods calculate a single average for each recording; the presence of this unexpected decrease may confound such estimates, leading to underestimation of indicators of resistance like the cerebrovascular resistance index (CVRi) (Panerai et al 2006). This unexpected finding may go some way to explaining the discrepancies in the existing literature concerning the effects of hypocapnia on dCA (Panerai 2003, Ainslie et al 2008). It was theorised that the temporary dysautoregulation observed may relate to changes in mental activation attributable to metronome inception, and the task of achieving and maintaining metronome-synchronised breathing to assess haemodynamic parameters adequately (Dineen et al 2010). The clinical benefits of minimisation of dysautoregulation are numerous and include improved ability to understand the pathophysiology of CA impairment in acute stroke but also provide more robust assessment of much needed interventions.

We therefore aimed to 1) investigate the effects of different hyperventilation strategies on temporal estimates of $\mathrm{dCA}$, with a view to validating the most effective strategy for inducing hypocapnia (herein referred to as 'induction strategy'); 2) investigate whether two different 
levels of hypocapnia $(-5 \mathrm{mmHg}$ and $-10 \mathrm{mmHg}$ from baseline) had differing effects during maintenance of hypocapnia on basic haemodynamic parameters (herein referred to as 'maintenance protocol').

\section{Methods}

\section{Study population}

The study was conducted in accordance with the Declaration of Helsinki (2000). Ethical approval was obtained from: 1) Induction strategies: Leicestershire, Northamptonshire and Rutland Research Ethics Committee (09/H0402/79); 2) Maintenance protocol: University of Leicester Ethics Committee (Reference: jm591-c033). In both cases, healthy volunteers were recruited from University departmental staff, students and their relatives. Participants aged above 18 years were included. Exclusion criteria were physical disease in the upper limb, poor insonation of both temporal bone windows and any significant history of cardiovascular, neurological or respiratory disease. All participants provided written, informed consent.

\section{Experimental protocol}

The research was undertaken in the University of Leicester's Cerebral Haemodynamics in Ageing and Stroke Medicine (CHiASM) research laboratory, maintained at a constant ambient temperature of approximately $24^{\circ} \mathrm{C}$ and free of distraction. For the purposes of the study, participants were asked to refrain from caffeine and alcohol in the 12-hour period prior to measurements being undertaken. Smokers were excluded. Beat-to-beat BP was recorded continuously using the Finometer ${ }^{\circledR}$ device (FMS, Finapres Measurement Systems, Arnhem, Netherlands), which was attached to the middle finger of the left hand. The servo-correcting mechanism of the Finometer ${ }^{\circledR}$ was switched on and then off prior to measurements. The hand bearing the finger cuff was at the level of the heart to negate any hydrostatic pressure artefact. Heart rate (HR) was recorded using a standard 3-lead electrocardiogram (ECG).

$\mathrm{EtCO}_{2}$ was measured throughout using small nasal cannulae (Salter Labs) connected to a capnograph (Capnocheck Plus). Bilateral insonation of the middle cerebral arteries (MCAs) was performed using transcranial Doppler (TCD) ultrasound (Viasys Companion III; Viasys Healthcare) with a $2 \mathrm{MHz}$ probe. This probe was secured in place with a head-frame that was 
adjusted to ensure comfort at the outset. The MCAs were identified according to two main characteristics: signal depth and velocities.

Measurements were continuously recorded at a rate of 500 samples/s in the PHYSIDAS data acquisition system (Department of Medical Physics, University Hospitals of Leicester). Systolic and diastolic brachial BP readings (OMRON Model 705IT) were performed at each stage of the measurements (normocapnia and hypocapnia) with a minimum of three recordings per individual. These values were then used to calibrate the Finometer recordings.

\section{Hyperventilation induction strategies:}

Following a 20-minute stabilisation period, a 5-minute baseline recording was taken of the subject breathing spontaneously at rest. Hyperventilation strategies were then repeated twice in random order, determined using a random number generator, with 5-minute intervals between each to allow stabilisation of all parameters and return to normocapnia. The different hyperventilation induction strategies are detailed below; hyperventilation being maintained for a minimum of 90 seconds in each strategy:

- Delayed metronome (hereafter, termed delayed): 2 minutes of spontaneous breathing followed by sudden inception of a metronome (KORG Metronome MA-30). The metronome speed was individualised to achieve a respiratory rate $40 \%$ greater than the original resting rate.

- Continuously increasing metronome (continuous): Use of a continuous metronome, started at a rate analogous to that of the subject's baseline resting rate. After one minute of baseline recording, the rate was increased gradually over a period of 60 seconds to reach a hyperventilation rate $40 \%$ greater than baseline.

- Voluntary (voluntary): Hyperventilation was induced without the use of a metronome. After 2 minutes of spontaneous respiration, subjects were vocally instructed to increase their respiratory rate independent to any other auditory stimulus.

For all the strategies, recordings were extended to include periods of spontaneous respiration for 2 minutes before and 3 minutes after hyperventilation in order to ascertain the temporal patterns of all parameters.

\section{Hyperventilation maintenance protocol:}

All measurements were conducted at a single visit. After a period of 5 minutes of stabilisation, participants performed a 5-minute baseline recording and then were asked to hyperventilate in 
random order, at different respiratory rates to produce incremental reductions in $\mathrm{EtCO}_{2}$ of $5 \mathrm{mmHg}$ and $10 \mathrm{mmHg}$ less than normocapnia for that individual. Hyperventilation was sustained for a minimum period of $60 \mathrm{~s}$. For hyperventilation, participants were asked to breathe with a metronome creating a respiratory rate of at least 5 breaths per minute above their resting rate for at least $90 \mathrm{~s}$ using the delayed metronome technique. Two-minute washout periods of normal respiration were allowed between successive measurements. Each incremental reduction in $\mathrm{EtCO}_{2}$ was repeated on two occasions during the same session.

\section{Data Analysis}

Data collected corresponded to individual files for each participant at baseline and during hypocapnia. First, data were inspected visually and calibrated to recorded systolic and diastolic OMRON BP. Narrow spikes $(<100 \mathrm{~ms})$ were removed using linear interpolation and the CBFV recording was then passed through a median filter. All signals were then low pass filtered with a zero-phase Butterworth filter with cut-off frequency of $20 \mathrm{~Hz}$. Automatic detection of the QRS complex of the ECG, to mark the R-R interval was used, but also visual inspection was undertaken with manual correction whenever necessary. This allowed mean $\mathrm{ABP}, \mathrm{HR}, \mathrm{EtCO}_{2}$ and mean CBFV to be calculated for each cardiac cycle. Randomisation was not disclosed until data collection was completed.

\section{Induction strategies and maintenance protocol}

Baseline files were analysed using a MW-ARMA model as described by Dineen et al (2010). Initially, an ARMA model is adopted to estimate the CBFV response to a step change in BP and the autoregulation index (ARI) is estimated by comparison with the 10 template CBFV step responses proposed by Tiecks et al (1995) using the first $60 \mathrm{~s}$ of data. The $60 \mathrm{~s}$ window is then shifted by $0.6 \mathrm{~s}$ and a new estimate of ARI is calculated. This process is then repeated until the end of the signal is reached thus generating ARI estimates at each $0.6 \mathrm{~s}$ period (Dineen et al 2010). This produced multiple estimates of ARI, which were then averaged to produce a single baseline ARI value for each file. Having estimates of ARI every $0.6 \mathrm{~s}$ is sufficient to represent changes that can take place due to hypocapnia, even at very high respiratory rates caused by hyperventilation, which produce estimates of $\mathrm{EtCO}_{2}$ with time intervals always longer than $2 \mathrm{~s}$. 
For the hyperventilation strategies, continuous estimates of ARI $(t)$ were produced for each file using the same MW-ARMA model. These were then digitally marked at the point of $\mathrm{EtCO}_{2}$ increase (signifying the end of hyperventilation) as this proved to be the most recognisable and reproducible point between strategies. Marked files were synchronised at 120 seconds and these data used to produce coherent averages of ARI(t) for each strategy, allowing comparison between the different conditions.

\section{Statistical analysis}

Data normality was confirmed with the Kolmogorov-Smirnov test. Baseline measurements were assessed for differences between values derived for right and left hemispheres using a paired Student's t-test. These were averaged when no significant differences were found.

For comparison of hyperventilation strategies, each participant acted as their own control, with an average value for each subject used where repeated manoeuvres were available for analysis. Hyperventilation strategies were compared using means of maximal changes in $\operatorname{ARI}(\mathrm{t})$ and $\mathrm{EtCO}_{2}(\mathrm{t})$. Two calculations of maximal ARI(t) change were performed. First, the difference between the initial baseline value and the minimal ARI during hyperventilation initiation was calculated. Additionally, the difference between this ARI trough and the subsequent peak of recovery was obtained. The maximal $\mathrm{EtCO}_{2}(\mathrm{t})$ change was calculated by subtracting the $\mathrm{EtCO}_{2}$ at maximal hypocapnia from an average baseline, calculated using the 30 seconds prior to each manoeuvre. All values were compared between the three strategies using repeated-measures ANOVA. Where statistical differences occurred, paired t-tests with a Bonferroni adjustment were employed to clarify where differences lay. Values of $\mathrm{p}<0.05$ were considered significant, giving an alpha significance level of $\mathrm{p}<0.017$ following Bonferroni adjustment.

\section{Results}

\section{Induction strategies}

Sixty-one healthy volunteers were recruited to the study (16 and 45 within the induction and maintenance protocol studies, respectively), one subject (from the induction strategies study) had all data removed from analysis as it failed to show a synchronous physiological reaction of BP or CBFV to $\mathrm{EtCO}_{2}$ changes. Demographic data for all 61 subjects are presented in Table 1. There were no significance differences between the male and female demographics. 
The mean ARI for the group was calculated as $5.95 \pm 1.07$ with a range of 4.36 to 7.86 during spontaneous respiration, which is within the normal range (Tiecks et al 1995, Carey et al 2000). Drifting of the BP signal led to rejection of two files, saturation of ARI was seen in a further two files and two manoeuvres failed to show a synchronous physiological reaction to changes in $\mathrm{EtCO}_{2}$. As such, the final statistical analysis included a total of 26 recordings for the delayed metronome strategy, 24 recordings for the continuous increasing metronome strategy and 25 recordings for the voluntary strategy.

Coherent averages of $\mathrm{ARI}(\mathrm{t})$ and $\mathrm{EtCO}_{2}(\mathrm{t})$ were produced for each strategy (Figure 1) and the means of maximal change calculated (Table 2). Significant differences were observed between strategies in both the initial ARI $(\mathrm{t})$ decrease and the ARI $(\mathrm{t})$ recovery. The extent of initial decrease was significantly smaller in the continuous metronome strategy compared to the delayed metronome and voluntary strategies $(\triangle \mathrm{ARI} 0.33 \pm 1.18,2.80 \pm 3.33$ and $3.69 \pm 2.79$ respectively, $\mathrm{p}<0.017$ ). There was no difference in the extent of initial decrease between the delayed metronome and voluntary strategies. The resultant degree of recovery needed to return the ARI to an augmented state was also significantly smaller in the continuous metronome when compared to the voluntary strategy $(\triangle \mathrm{ARI} 1.98 \pm 1.90$ and $4.79 \pm 2.94$ respectively, $\mathrm{p}<0.017$ ); however, the difference between the continuous and delayed metronome strategies was borderline after the Bonferroni correction $(\mathrm{p}=0.02)$.

Differences were also observed in baseline $\mathrm{EtCO}_{2}$ and in the extent of induced hypocapnia between strategies. Baseline $\mathrm{EtCO}_{2}$ was significantly lower in the continuous metronome strategy compared to the delayed metronome and voluntary strategies $\left(\mathrm{EtCO}_{2} 34.5 \pm 2.3,39.02\right.$ \pm 1.6 and $38.6 \pm 2.0 \mathrm{mmHg}$ respectively, $\mathrm{p}<0.017$ ). The degree of induced hypocapnia was similar between the delayed metronome and continuous metronome strategies $\left(\Delta \mathrm{EtCO}_{2} \quad-5.5\right.$ \pm 3.3 and $-4.8 \pm 2.3 \mathrm{mmHg}$, respectively, $\mathrm{p}=0.25)$, but significantly greater with the voluntary strategy $\left(\Delta \mathrm{EtCO}_{2}-8.3 \pm 3.6 \mathrm{mmHg}, \mathrm{p}<0.017\right)$.

In addition, coherent average graphs were also created for $\operatorname{CBFV}(\mathrm{t})$, critical closing pressure $(\mathrm{CrCP})(\mathrm{t})$, resistance-area product $(\mathrm{RAP})(\mathrm{t}), \mathrm{ABP}(\mathrm{t})$ and $\mathrm{HR}(\mathrm{t})$. These graphs allowed visual inspection of the stability of each parameter throughout the strategies (Figures 2 - 6). Across all parameters, the visual depictions demonstrated greater parameter stability over time with the continuous metronome strategy as compared to the two alternatives. The delayed and voluntary metronome strategies demonstrated more abrupt changes at the beginning and end of the measurement periods. 
281 The maintenance of hypocapnia protocol involved two incremental reductions in $\mathrm{EtCO}_{2}$ during

282 the same session. These were randomised and involved a significant washout period. As expected, the targeted reductions in $\mathrm{EtCO}_{2}$ to $-5 \mathrm{mmHg}$ and $-10 \mathrm{mmHg}$ were accompanied by increases in respiratory rate (Table 1). Interestingly, despite significant differences in $\mathrm{EtCO}_{2}$ during each incremental lowering $(\mathrm{p}<0.0001)$, only CBFV $(\mathrm{p}=0.1381)$ remained unchanged as ABP $(p<0.0001)$ and HR $(p=0.0009)$ both demonstrated significant differences in response to the step change in hyperventilation (Figure 7).

\section{Discussion}

Use of a continuous metronome to induce hypocapnia rather than the sudden inception of an auditory stimulus appeared to reduce the initial decrease in autoregulatory capacity seen previously in work by Dineen et al (2010). This is important as it afforded better estimates of $\mathrm{CA}$, especially when using original batch-processing analysis techniques to calculate mean values of ARI for an entire file. Furthermore, maintenance of $\mathrm{EtCO}_{2}$ at differing levels of targeted hypocapnia $(-5 \mathrm{mmHg}$ and $-10 \mathrm{mmHg})$ demonstrated no significant effect on central haemodynamic parameters (CBFV), but affected peripheral parameters including HR and ABP. The authors are not aware of any other studies investigating the effect of hyperventilation protocols on continuous estimates of dCA.

This advancement in understanding not only provides important perspectives on the responsiveness of the cerebral circulation to incremental hypocapnia, but also provides a strong rationale for continuous metronome use in clinical studies assessing cerebral haemodynamics in acute stroke. There has been a significant paucity of confirmatory studies designed to provide a robust assessment of hypocapnia and CA particularly with reference to the variety of potential physiological measurement approaches (Minhas et al 2018b).

The coherent averages of ARI(t) (Figure 1) clearly demonstrated a reduced initial ARI decrease using the continuous increasing metronome protocol compared to the original delayed metronome. This observation is supported by statistical analysis, which showed an ARI drop of $0.33 \pm 1.18$ for the continuous metronome protocol and $2.80 \pm 3.33$ for the delayed metronome $(p<0.017)$. The smaller standard deviation for the continuous metronome protocol also highlighted the greater stability of these measurements compared to those obtained using 
the original protocol. The minimised initial drop is also reflected in the smaller degree of recovery required to return the ARI to an augmented state (Figure 1). Although not statistically significant, when a single fiducial point is selected, the difference in degree of recovery is clearly visible when examining the complete temporal pattern of the coherent average graphs (Figures 1-6). In addition, the greater stability demonstrated by the $\operatorname{CBFV}(\mathrm{t}), \operatorname{CrCP}(\mathrm{t}), \operatorname{RAP}(\mathrm{t})$, $\operatorname{ABP}(t)$ and $\operatorname{HR}(t)$ coherent averages from the continuous metronome condition provided further evidence of the advantages of this protocol over the original (Figures 2-6).

Visual inspection shows the augmentation of $\mathrm{ARI}(\mathrm{t})$ diminished prior to the cessation of hyperventilation in the continuous metronome condition (Figure 1). This differs to the pattern of $\mathrm{ARI}(\mathrm{t})$ in both the delayed metronome and voluntary protocols, where $\mathrm{dCA}$ remained augmented until hyperventilation was discontinued. On examination of the original data this was found to be a real effect, with the subjects divided into two groups: those who maintained ARI throughout hypocapnia and those who did not. Whilst the continuous metronome protocol appears to predominantly remove what can be thought of as an "alert reaction" in response to hyperventilation induction, it is possible that it induces a less severe but more prolonged mental stress effect. Therefore, rather than producing a sudden initial decrease in ARI, the prolonged mental activation competes with the usual hypocapnic augmentation of dCA. The high metronome rate and long duration of synchronised breathing may have caused a degree of mental and physical fatigue towards the end of measurement that is reflected by a premature ARI decrease in selected individuals. However, the maintenance protocol demonstrated a steady $\mathrm{EtCO}_{2}, \mathrm{HR}$ and ABP throughout with little significant variation in trend. This is crucial when you consider the high chance of fatigue in an acute stroke population and demonstrates a strong rationale for use of the maintenance protocol to ensure more accurate peripheral and cerebral haemodynamic assessment. This is particularly the case as cerebral haemodynamic parameters including RAP and $\mathrm{CrCP}$ behaved as expected through hypocapnia influenced change on cerebrovascular resistance and intracranial pressure, respectively.

The voluntary protocol was designed to investigate the physiological effect of hypocapnia on dCA without the stress of synchronising breathing to a metronome. For this reason, it was expected that it would cause less of an initial ARI decrease than the delayed metronome protocol. However, on commencement of voluntary hyperventilation, a large decrease in the autoregulatory capacity occurs that is comparable to that of the delayed metronome $(p=0.30)$. This may have occurred for several reasons. The sudden interception of vocal instruction may have startled some participants, creating an 'alert reaction' similar to that of the metronome 
inception. A further possibility is that, without a metronome, participants overcompensated to ensure hyperventilation, meaning mental stimulation and respiratory effort were greater during this protocol. This is supported by a larger $\mathrm{EtCO}_{2}$ decrease in the voluntary condition compared to the other protocols (Figure 1).

The continuous metronome protocol induced a comparable degree of hypocapnia to that of the delayed metronome protocol supporting implementation of the new protocol as the hypocapnia induced was of a similar magnitude to that obtained with the previous method, making it a suitable alternative for assessment of CVRi.

Baseline $\mathrm{EtCO}_{2}$ was significantly lower in the continuous metronome protocol than in the delayed metronome or voluntary protocols. In the latter two protocols, baseline $\mathrm{EtCO}_{2}$ was calculated using the initial period of spontaneous respiration; this was observed to be naturally erratic in most participants, with temporary periods of very low respiratory rate. In contrast, participants synchronised their breathing from the beginning of the continuous metronome recordings and consequently had no opportunity to retain $\mathrm{CO}_{2}$ through decreases in respiratory rate. This explained the lower values of baseline $\mathrm{EtCO}_{2}$ for that protocol. These findings apply to the maintenance protocol as the method used was analogous with the delayed protocol and although increments were close to targets of $-5 \mathrm{mmHg}$ and $-10 \mathrm{mmHg}$ below baseline, lower values may have been achieved had a continuous protocol been employed prior to maintenance. Ideally, performing the study in a ventilated population would have avoided variation in baseline $\mathrm{EtCO}_{2}$ between groups and hence provided further information on the extent to which smaller changes in the continuous metronome strategy are explained by lower baseline $\mathrm{EtCO}_{2}$ values in this group.

In summary, this study provides strong evidence for clinical studies assessing hypocapnic interventions to utilise a continuous metronome protocol to minimise dysautoregulation and hence better understand the pathophysiological changes associated with hypocapnia in clinical settings including acute stroke and during anaesthesia, settings in which such interventions have been assessed and continue to be assessed.

\section{Limitations}

Individual differences in baseline autoregulatory capacity ought not to have biased comparisons made between protocols as every participant was represented equally between the conditions. In addition, each subject acted as their own control across the protocols, with 
participants being excluded from the final analysis if data were missing from any of the conditions.

Although small, the sample size for the study had adequate power to detect the $\triangle \mathrm{ARI}$ differences between protocols. A study by Brodie et al (2009) assessed the reproducibility of ARI measurements and determined that in order to achieve $80 \%$ statistical power at the $p=0.05$ significance level, a sample size of 11 was necessary to detect a change in ARI of 2 (Brodie et al 2009). The sample size in this study was 15 and the difference in the initial $\Delta$ ARI between the continuous and delayed metronome condition was +2.47 . Whilst it is recognised that the study by Brodie et al (2009) evaluated ARI calculations based on the traditional batchprocessing method, the greater sensitivity of the MW-ARMA model (Panerai 2003) should if anything increase the power of this study. However, both physiological and technical limitations led to differing numbers of files for each group being included in the final analysis. Although the study was overpowered to detect the differences discussed, future studies in larger numbers with imbalanced groups may benefit from hierarchical or mixed-effects model statistical approaches.

Synchronising the files to produce coherent averages for the continuous increasing metronome condition was challenging. In the study by Dineen et al (2010) recordings were synchronised by the transient rise in CBFV signifying the beginning of hyperventilation (Dineen et al 2010). However, with the continuous metronome protocol this CBFV peak does not occur, and therefore recordings for all protocols were marked by the end of hyperventilation using the $\mathrm{EtCO}_{2}$ trace, this being the most reproducible point between files. Marking and synchronisation of data should ideally occur at the point of hyperventilation initiation to increase accuracy and reliability of the averaged data. It may be possible in future work to digitally mark the start of hyperventilation on the traces at time of recording, thus avoiding this problem.

There are also some practical considerations for implementation of the continuous metronome protocol. During the condition, participants were required to breathe in time with a metronome for a total of four minutes. Whilst most subjects were able to synchronise their breathing at baseline, some patients were unable to maintain this throughout the rate increase. The speed of the metronome may also have been stressful for the participant due to technical problems encountered when trying to adjust the metronome speed manually. This problem could be overcome with the use of a programmable digital metronome rather than the use of a hand-held device. This measurement error will have contributed to the shortfall in the difference (2- 
$3 \mathrm{mmHg})$ between target incremental levels $\left(-5 \mathrm{mmHg}\right.$ and $\left.-10 \mathrm{mmHg} \mathrm{EtCO}_{2}\right)$. However, despite generating an adequate increment, $\mathrm{CBFV}$ values did not differ, therefore questioning the value of multiple levels of hypocapnic measurement. This is crucial as studies have previously used multiple increments to assess cerebral haemodynamic change, and although these have provided valuable information on the relationship of $\mathrm{EtCO}_{2}$ change on $\mathrm{dCA}$, future studies may not require multiple increments unless significantly greater than $3 \mathrm{mmHg}$ (Ainslie et al 2008, Minhas et al 2018b). Therefore, there remains a possibility that had the increment been larger, CBFV may have differed, though we can conclude that an increment of $2-3 \mathrm{mmHg}$ does not demonstrate significant CBFV change to warrant such increments in the study design. Furthermore, the authors are aware that ventilation is influenced by breathing frequency and tidal volume. In this investigation, the authors only manipulated breathing frequency to induce hyperventilation and thus hypocapnia. We do not report any data for tidal volume though the authors acknowledge this would be of potential value.

Another consideration is that whilst some individuals were confident synchronising their breathing to an external stimulus, those who were not, seemed to find it a particular challenge when the stimulus was varying. In this study the problem was relatively minor; however the applicability of the continuous metronome protocol must be tested in other populations. Of particular concern are the elderly and individuals of ill health who may have limited concentration or lower tolerance for sustained hypocapnia. Furthermore, sex-related differences in cerebral haemodynamics have been demonstrated though underlying mechanisms remain unclear with vessel diameter, hormonal variation and basic metabolic rate all discussed as influencing factors (Deegan et al 2009). However, female subjects have higher MCA velocities, $\mathrm{CO}_{2}$ cerebrovascular reactivity and vasomotor range than males (Deegan et al 2009, Minhas et al 2018c). This study was not designed to inform the extent to which sex may influence the initiation and maintenance of hyperventilatory interventions, though it is reassuring the baseline demographic data did not demonstrate sex differences.

As with any study of this kind, the limitations of TCD for measuring CA have to be considered. The use of TCD to estimate CBF relies upon the assumption that the MCA has a fixed diameter and therefore any changes in measured CBFV correspond to changes in flow. Numerous studies have assessed MCA diameter change during changes in $\mathrm{ABP}$ and $\mathrm{EtCO}_{2}$. The majority of these studies have demonstrated during direct visualisation of both the MCA and internal carotid artery by MRI assessment that no change in diameter occurs (Giller et al 1993, Newell et al 1994, Serrador et al 2000). However, more recent studies have demonstrated that during 
high levels of hypercapnia there is sensitivity to $\mathrm{CO}_{2}$ change and hence diameter change

441 (Verbree et al 2014, Coverdale et al 2014). Importantly, there remains a paucity of data supporting the existence of such changes in hypocapnic conditions. Therefore, for the purposes of this study the changes in CBFV measured were assumed to correspond to changes in $\mathrm{CBF}$.

\section{Future directions}

445 Further work investigating the independent physiological effects of mental stimulation and changing $\mathrm{PaCO}_{2}$ on $\mathrm{dCA}$ would be of value. Investigation into the effects of brain activation could be achieved by sudden synchronisation of breathing to an external stimulus set at an identical rate to the participants' resting respiratory rate. The effect of changing $\mathrm{PaCO}_{2}$ could be assessed using a re-breathing protocol to passively alter the concentration of inspired gases.

450 This approach has been previously used in autoregulation studies but in combination with a MW-ARMA model, it may extend our understanding of the physiological parameters affecting CA.

\section{Conclusions}

455

In conclusion, use of a continuous metronome to induce hypocapnia rather than the sudden inception of an auditory stimulus appears to reduce the initial decrease in autoregulatory capacity seen previously in work by Dineen et al (2010). This is important as it affords better estimates of CA, especially if using original batch-processing analysis techniques to calculate mean values of ARI for an entire recording. Dysautoregulation can be minimised by continuous metronome use during hyperventilation-induced hypocapnia. This advancement in understanding of the behaviour of CA during hypocapnia permits safer delivery of CA targeted interventions, particularly in neurologically vulnerable patient populations.

\section{Competing interests}

No competing interests 
468 JSM is Dunhill Medical Trust Clinical Research Training Fellow (RTF97/0117) at the

469 Department of Cardiovascular Sciences, University of Leicester. TGR is an NIHR Senior

470 Investigator. We thank the subjects for their willingness to participate. This work falls under

471 the portfolio of research conducted within the NIHR Leicester Biomedical Research Centre.

472

473 Author Contribution Statement

474 RBP and TGR conceived and designed the research. JSM and CK conducted experiments.

475 JSM, CK and RBP analysed the data. JSM, CK, RBP and TGR wrote the manuscript. All 476 authors read and approved the manuscript. 


\section{References}

Aaslid R, Lindegaard KF, Sorteberg W and Nornes H 1989 Cerebral autoregulation dynamics in humans Stroke 20 45-52

Ainslie PN, Celi L, McGrattan K, Peebles K and Ogoh S 2008 Dynamic cerebral autoregulation and baroreflex sensitivity during modest and severe step changes in arterial $\mathrm{PCO}_{2}$ Brain. Res. 1230 $115-124$

Battisti-Charbonney A, Fisher J and Duffin J 2011 The cerebrovascular response to carbon dioxide in humans J.Physiol. 589 3039-3048

Birch AA, Dirnhuber MJ, Hartley-Davies R, lannotti F and Neil-Dwyer G 1995 Assessment of autoregulation by means of periodic changes in blood pressure Stroke 26 834-837

Brodie FG, Atkins ER, Robinson TG and Panerai RB 2009 Reliability of dynamic cerebral autoregulation measurement using spontaneous fluctuations in blood pressure Clin.Sci. 116 513-520

Carey BJ, Eames PJ, Blake MJ, Panerai RB and Potter JF 2000 Dynamic cerebral autoregulation is unaffected by aging Stroke 31 2895-2900

Coverdale NS, Gati JS, Opalevych O, Perrotta A and Shoemaker JK 2014 Cerebral blood flow velocity underestimates cerebral blood flow during modest hypercapnia and hypocapnia J.Appl.Physiol. 117 1090-1096

Deegan BM, Sorond FA, Lipsitz LA, Olaighin G and Serrador JM 2009 Gender related differences in cerebral autoregulation in older healthy subjects Conf.Proc.IEEE.Eng.Medicine.Biol.Soc. 28592862

Dineen NE, Brodie FG, Robinson TG and Panerai RB 2010 Continuous estimates of dynamic cerebral autoregulation during transient hypocapnia and hypercapnia J.Appl.Physiol. 108 604-613

Eames PJ, Potter JF and Panerai RB 2004 Influence of controlled breathing patterns on cerebrovascular autoregulation and cardiac baroreceptor sensitivity Clin.Sci. 106 155-162

Giller CA, Bowman G, Dyer H, Mootz L and Krippner W 1993 Cerebral arterial diameters during changes in blood pressure and carbon dioxide during craniotomy Neurosurgery 32 737-42

Ide K, Eliasziw M and Poulin MJ 2003 Relationship between middle cerebral artery blood velocity and end-tidal $\mathrm{PCO}_{2}$ in the hypocapnic-hypercapnic range in humans J.Appl.Physiol. 95 129-137

Lassen NA 1959 Cerebral blood flow and oxygen consumption in man Physiol.Rev. 39 183-238

Long B, Koyfman A and Vivirito MA 2017 Capnography in the Emergency Department: A Review of Uses, Waveforms, and Limitations J.Emerg.Med. 53 829-842

Ma X, Willumsen L, Hauerberg J, Pedersen DB and Juhler M 2000 Effects of graded hyperventilation on cerebral blood flow autoregulation in experimental subarachnoid hemorrhage J.Cereb.Blood Flow Metab. 20 718-725 
Markus HS and Harrison MJ 1992 Estimation of cerebrovascular reactivity using transcranial Doppler, including the use of breath-holding as the vasodilatory stimulus Stroke $\mathbf{2 3}$ 668-673

McCulloch TJ, Boesel TW and Lam AM 2005 The effect of hypocapnia on the autoregulation of cerebral blood flow during administration of isoflurane Anesth.Analg 100 1463-7

Minhas JS 2018 Highlighting the Potential Value of Capnography in Acute Stroke J.Emerg.Med. 55 130-131

Minhas JS, Panerai RB, Ghaly G, Divall P and Robinson TG 2018 Cerebral autoregulation in hemorrhagic stroke: A systematic review and meta-analysis of transcranial Doppler ultrasonography studies J.Clin.Ultrasound 10.1002/jcu.22645

Minhas JS, Panerai RB and Robinson TG 2018a Feasibility of Improving Cerebral Autoregulation in Acute Intracerebral Haemorrhage (BREATHE-ICH) study: a protocol for an experimental interventional study BMJ Open 8 e020758-2017-020758

Minhas JS, Panerai RB and Robinson TG 2018b Modelling the cerebral haemodynamic response in the physiological range of $\mathrm{PaCO}_{2}$ Phys. Meas. 39 065001-6579/aac76b

Minhas JS, Panerai RB and Robinson TG 2018c Sex differences in cerebral haemodynamics across the physiological range of $\mathrm{PaCO}_{2}$ Phys.Meas. 39105009

Newell DW, Aaslid R, Lam A, Mayberg TS and Winn HR 1994 Comparison of flow and velocity during dynamic autoregulation testing in humans Stroke 25 793-797

Newell DW, Weber JP, Watson R, Aaslid R and Winn HR 1996 Effect of transient moderate hyperventilation on dynamic cerebral autoregulation after severe head injury Neurosurgery 39 35-44

Ogoh S, Hayashi N, Inagaki M, Ainslie PN and Miyamoto T 2008 Interaction between the ventilatory and cerebrovascular responses to hypo- and hypercapnia at rest and during exercise J.Appl.Physiol. 586 4327-4338

Ogoh S, Nakahara H, Ainslie PN and Miyamoto T 2010 The effect of oxygen on dynamic cerebral autoregulation: critical role of hypocapnia J.Appl.Physiol. 108 538-543

Panerai RB 2008 Cerebral autoregulation: from models to clinical applications Cardiovasc. Eng. 8 4259

Panerai RB 2003 The critical closing pressure of the cerebral circulation Med.Eng.Phys. 25 621-632

Panerai RB, Deverson ST, Mahony P, Hayes P and Evans DH 1999 Effects of $\mathrm{CO}_{2}$ on dynamic cerebral autoregulation measurement Phys.Meas. 20 265-275

Panerai RB, Eames PJ and Potter JF 2006 Multiple coherence of cerebral blood flow velocity in humans Am.J.Physiol.Heart.Circ. Physiol. 291 H251-9

Patel N, Panerai RB, Haunton V, Katsogridakis E, Saeed NP, Salinet A, Brodie F, Syed N, D'Sa S and Robinson TG 2016 The Leicester cerebral haemodynamics database: normative values and the influence of age and sex Phys.Meas. 37 1485-1498 
Paulson OB, Strandgaard S and Edvinsson L 1990 Cerebral autoregulation Cereb.Brain Metab.Rev 2 161-192

Rittayamai N, Tscheikuna J and Rujiwit P 2014 High-flow nasal cannula versus conventional oxygen therapy after endotracheal extubation: a randomized crossover physiologic study Resp.Care. $\mathbf{5 9}$ 485-490

Royal College of Physicians Intercollegiate Stroke Working Party 2016 National clinical guideline for stroke. Available: https://www.rcplondon.ac.uk/guidelines-policy/stroke-guidelines [2017, 15/08]

Serrador JM, Picot PA, Rutt BK, Shoemaker JK and Bondar RL 2000 MRI measures of middle cerebral artery diameter in conscious humans during simulated orthostasis Stroke 31 1672-1678

Strauss GI 2007 The effect of hyperventilation upon cerebral blood flow and metabolism in patients with fulminant hepatic failure Dan.Med.Bull. 54 99-111

Takahashi C, Liang CW, Liebeskind DS and Hinman JD 2014 To Tube or Not to Tube? The Role of Intubation during Stroke Thrombectomy Front.Neurol. 5170

Tiecks FP, Lam AM, Aaslid R and Newell DW 1995 Comparison of static and dynamic cerebral autoregulation measurements Stroke 26 1014-1019

Verbree J, Bronzwaer AS, Ghariq E, Versluis MJ, Daemen MJ, van Buchem MA, Dahan A, van Lieshout $\mathrm{JJ}$ and van Osch MJ 2014 Assessment of middle cerebral artery diameter during hypocapnia and hypercapnia in humans using ultra-high-field MRI J.Appl.Physiol. 117 1084-1089 
Table 1. Population characteristics and baseline parameter values

\begin{tabular}{|c|c|c|c|c|c|c|}
\hline & \multicolumn{3}{|c|}{ Induction Protocol } & \multicolumn{3}{|c|}{ Maintenance Protocol } \\
\hline & Male & Female & p-value & Male & Female & p-value \\
\hline Number (n) & 7 & 8 & - & 19 & 26 & - \\
\hline Age (years) & $\begin{array}{l}37 \pm 15 \\
{[21,62]}\end{array}$ & $\begin{array}{l}33 \pm 12 \\
{[22,53]}\end{array}$ & 0.57 & $\begin{array}{l}36 \pm 15 \\
{[21,69]}\end{array}$ & $\begin{array}{l}39 \pm 14 \\
{[21,69]}\end{array}$ & 0.87 \\
\hline Systolic BP (mmHg) & $\begin{array}{c}127 \pm 18 \\
{[100,146]}\end{array}$ & $\begin{array}{c}117 \pm 15 \\
{[100,144]}\end{array}$ & 0.67 & $\begin{array}{c}125 \pm 12 \\
{[106,146]}\end{array}$ & $\begin{array}{l}118 \pm 15 \\
{[91,151]}\end{array}$ & 0.07 \\
\hline Diastolic BP (mmHg) & $\begin{array}{c}81 \pm 18 \\
{[56,107]}\end{array}$ & $\begin{array}{c}70 \pm 12 \\
{[57,96]}\end{array}$ & 0.26 & $\begin{array}{c}72 \pm 8 \\
{[54,90]}\end{array}$ & $\begin{array}{l}70 \pm 11 \\
{[47,96]}\end{array}$ & 0.52 \\
\hline $\mathrm{HR}(\mathrm{bpm})$ & $\begin{array}{l}64 \pm 11 \\
{[54,82]}\end{array}$ & $\begin{array}{c}65 \pm 9 \\
{[57,79]}\end{array}$ & 0.67 & $\begin{array}{c}66 \pm 13 \\
{[49,101]}\end{array}$ & $\begin{array}{l}72 \pm 10 \\
{[59,97]}\end{array}$ & 0.19 \\
\hline $\mathrm{RR}\left(\min ^{-1}\right)$ baseline & $\begin{array}{c}13 \pm 3 \\
{[10,20]}\end{array}$ & $\begin{array}{c}14 \pm 2 \\
{[10,16]}\end{array}$ & 0.34 & $\begin{array}{c}15 \pm 3 \\
{[10,20]}\end{array}$ & $\begin{array}{c}15 \pm 3 \\
{[10,20]}\end{array}$ & 0.62 \\
\hline $\mathrm{RR}\left(\mathrm{min}^{-1}\right)-5 \mathrm{mmHg}$ & & & & $\begin{array}{c}21 \pm 1 \\
{[20,24]}\end{array}$ & $\begin{array}{c}21 \pm 2 \\
{[16,25]}\end{array}$ & 0.53 \\
\hline $\mathrm{RR}\left(\mathrm{min}^{-1}\right)-10 \mathrm{mmHg}$ & & & & $\begin{array}{c}28 \pm 1 \\
{[26,31]}\end{array}$ & $\begin{array}{c}27 \pm 2 \\
{[23,34]}\end{array}$ & 1.00 \\
\hline
\end{tabular}

570

571

572

573

574

575

576

Values are given as the mean \pm standard deviation with the range denoted in brackets. BP, blood pressure; HR, heart rate; RR, respiratory rate.

Table 2. Mean values of ARI for each protocol at baseline and showing the means of maximal changes. Baseline $\mathrm{EtCO}_{2}$ and the extent of induced hypocapnia is also shown.

\begin{tabular}{|l|l|c|c|c|c|}
\hline \multicolumn{2}{|c|}{} & $\begin{array}{c}\text { Delayed } \\
\text { metronome }\end{array}$ & $\begin{array}{c}\text { Continuous } \\
\text { metronome }\end{array}$ & Voluntary & P-value \\
\hline \multirow{4}{*}{ ARI } & Baseline & $\begin{array}{c}5.62 \\
\pm 1.78\end{array}$ & $\begin{array}{c}5.66 \\
\pm 2.04\end{array}$ & $\begin{array}{c}6.12 \\
\pm 1.74\end{array}$ & 0.65 \\
\cline { 2 - 6 } & Extent of initial decrease & 2.80 & 0.33 & 3.69 & $\mathbf{0 . 0 0 1 *}$ \\
& & \pm 3.33 & \pm 1.18 & \pm 2.79 & \\
\cline { 2 - 6 } & Degree of recovery & 4.08 & 1.98 & 4.79 & $\mathbf{0 . 0 1}^{*}$ \\
& & \pm 2.57 & \pm 1.90 & \pm 2.94 & \\
\hline \multirow{3}{*}{ EtCO } & & 39.0 & 34.5 & 38.6 & $<\mathbf{0 . 0 0 1}^{*}$ \\
$(\mathrm{mmHg})$ & Baseline & \pm 1.6 & \pm 2.3 & \pm 2.0 & \\
\cline { 2 - 6 } & & 5.5 & 4.8 & 8.3 & $<\mathbf{0 . 0 0 1 ^ { * }}$ \\
\hline
\end{tabular}

Values are given as the mean \pm standard deviation. Significant differences between the protocols are marked in using *. ARI, autoregulation index; $\mathrm{EtCO}_{2}$, end-tidal arterial pressure of carbon dioxide. 


\section{$579 \quad$ Figure legends}

580 Figure 1. Coherent average graphs of $\mathrm{ARI}(\mathrm{t})$ and $\mathrm{EtCO}_{2}(\mathrm{t})$ for each protocol.

581 Error bars show \pm one SEM, calculated using the maximum standard deviation and displayed

582 at the point where maximum error occurred

583 Figure 2. Coherent average graphs of $\mathrm{CBFV}(\mathrm{t})$ for each protocol

584 Error bars show \pm one SEM, calculated using the maximum standard deviation and displayed 585 at the point where maximum error occurred

586 Figure 3. Coherent average graphs of critical closing pressure $(\mathrm{CrCP})(\mathrm{t})$ for each protocol

587 Error bars show \pm one SEM, calculated using the maximum standard deviation and displayed 588 at the point where maximum error occurred

589 Figure 4. Coherent averages of resistance-area product (RAP)(t) for each protocol

590 Error bars show \pm one SEM, calculated using the maximum standard deviation and displayed 591 at the point where maximum error occurred

592

$593 \quad$ Figure 5. Coherent averages of $\mathrm{ABP}(\mathrm{t})$ for each protocol

594 Error bars show \pm one SEM, calculated using the maximum standard deviation and displayed 595 at the point where maximum error occurred

$597 \quad$ Figure 6. Coherent averages of HR(t) for each protocol

598 Error bars show \pm one SEM, calculated using the maximum standard deviation and displayed 599 at the point where maximum error occurred

600 Figure 7. Coherent averages of $\mathrm{ABP}(\mathrm{t}), \mathrm{HR}(\mathrm{t}), \mathrm{CBFV}(\mathrm{t})$ and $\mathrm{EtCO}_{2}(\mathrm{t})$ for the maintenance 601 protocol

602 Error bars show \pm one standard deviation. Values of $\mathrm{p}<0.05$ were considered significant. 


\section{Figures:}

Figure 1: Coherent average of $\mathrm{ARI}(\mathrm{t})$ and $\mathrm{EtCO}_{2}(\mathrm{t})$ for each hyperventilation strategy for induction of hypocapnia.
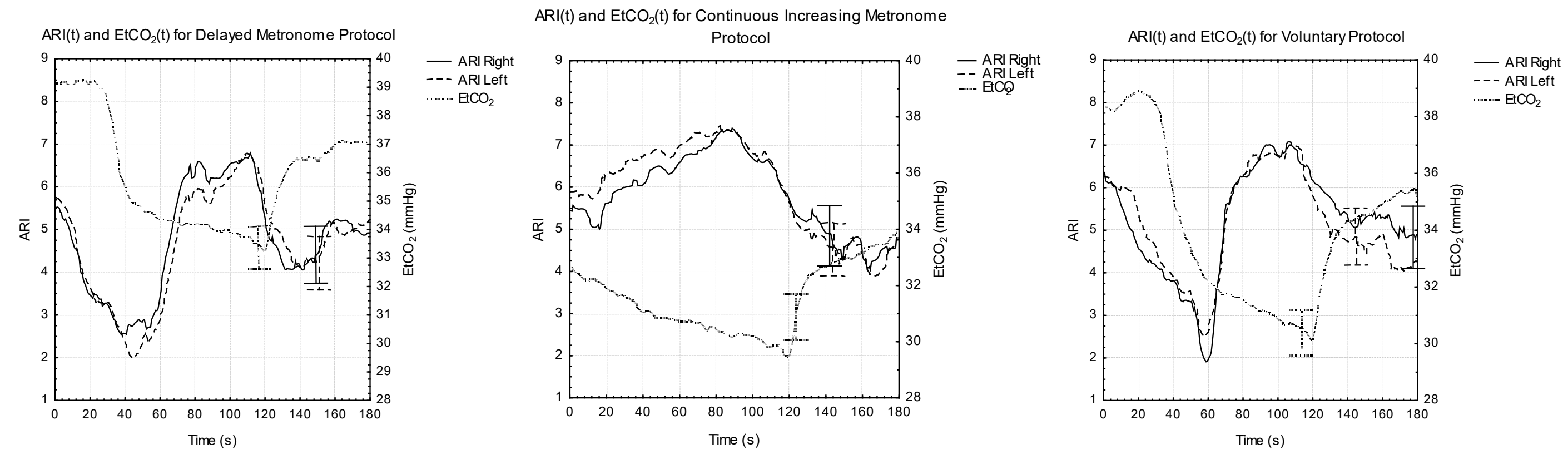

Error bars show \pm one SEM, calculated using the maximum standard deviation and displayed at the point where maximum error occurred 
Figure 2: Coherent average graphs of $\mathrm{CBFV}(\mathrm{t})$ for each hyperventilation strategy for induction of hypocapnia
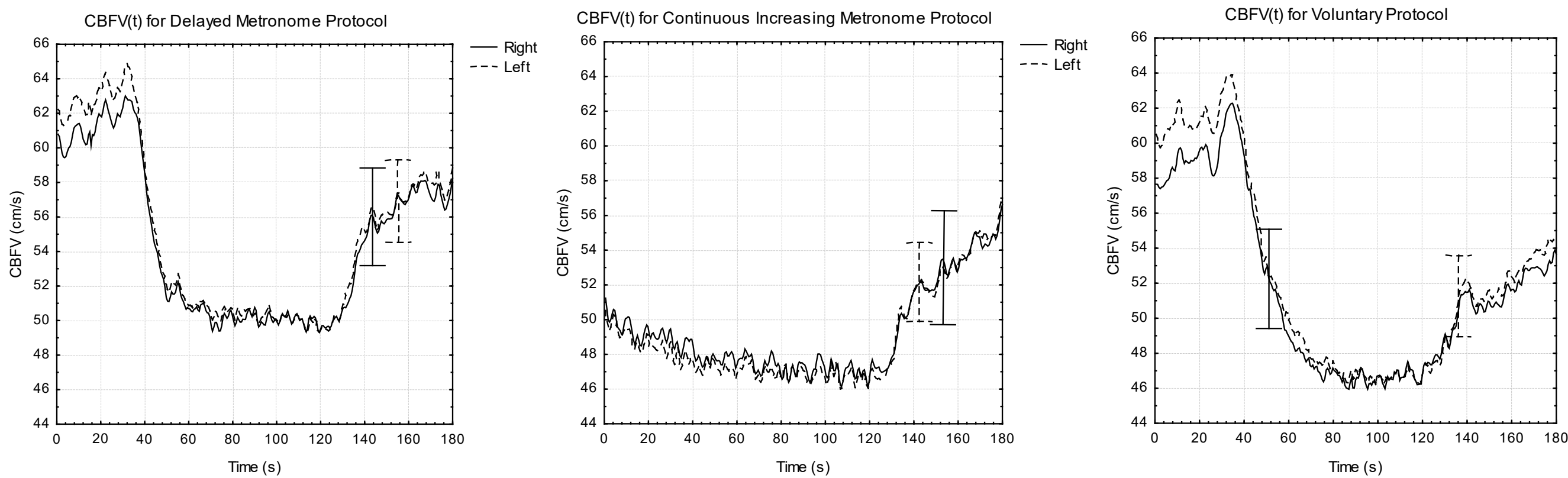

Error bars show \pm one SEM, calculated using the maximum standard deviation and displayed at the point where maximum error occurred 


\section{Figure 3: Coherent average graphs of critical closing pressure (CrCP)(t) for each protocol}
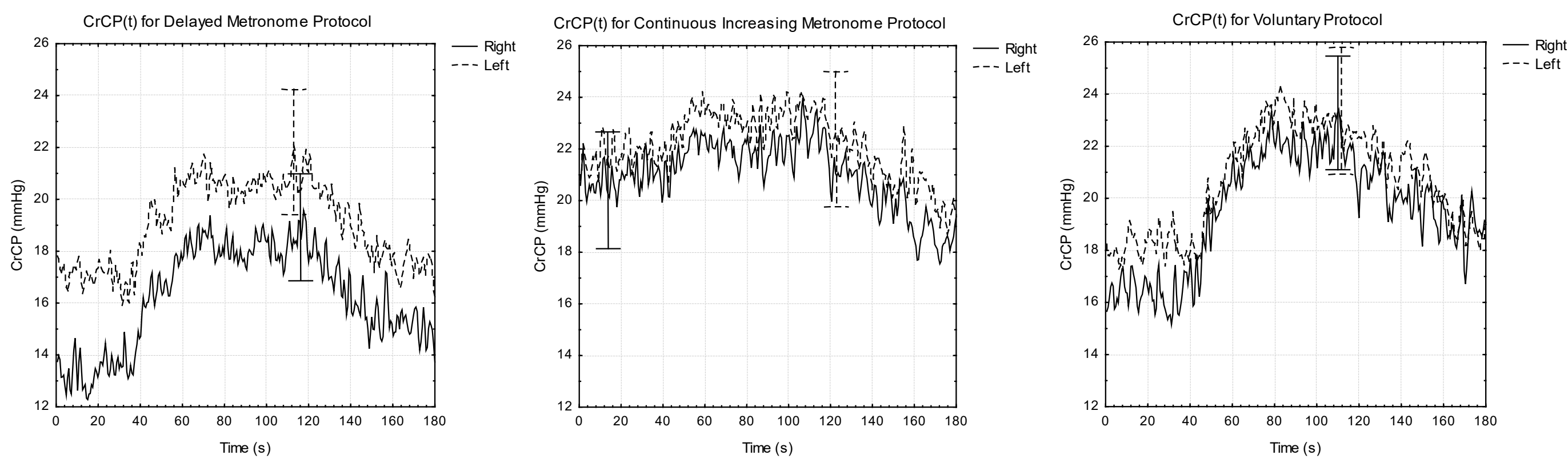

Error bars show \pm one SEM, calculated using the maximum standard deviation and displayed at the point where maximum error occurred 

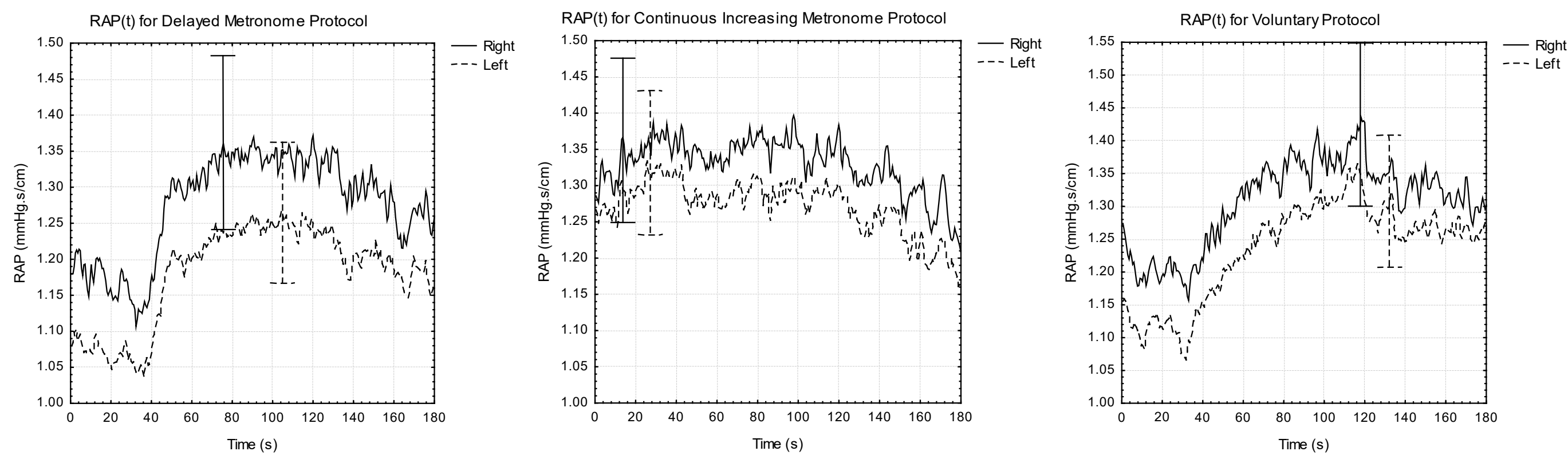

Error bars show \pm one SEM, calculated using the maximum standard deviation and displayed at the point where maximum error occurred 


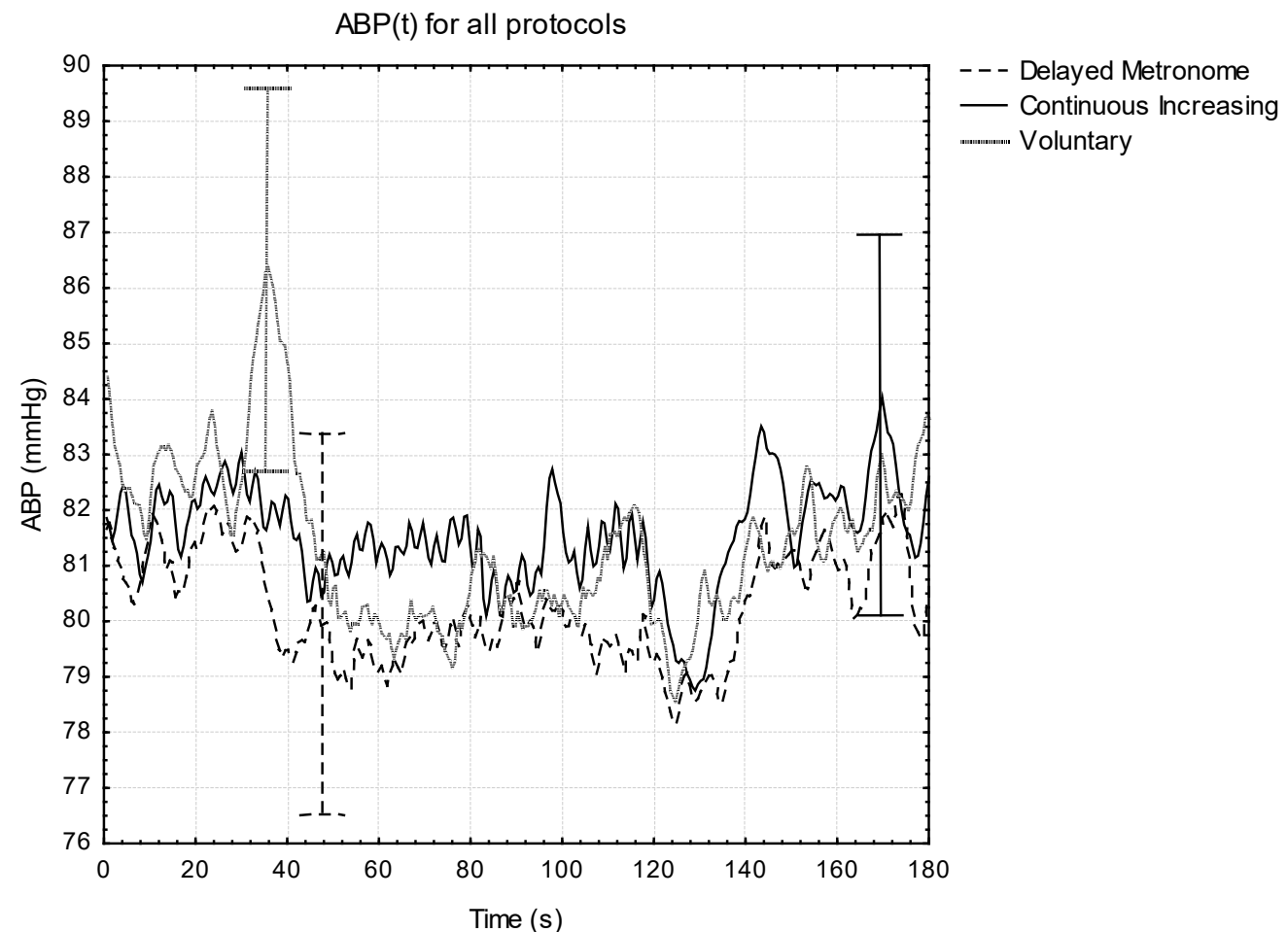

3

Error bars show \pm one SEM, calculated using the maximum standard deviation and displayed at the point where maximum error occurred

Figure 6: Coherent averages of $\mathrm{HR}(\mathrm{t})$ for each hyperventilation strategy for induction of hypocapnia 8

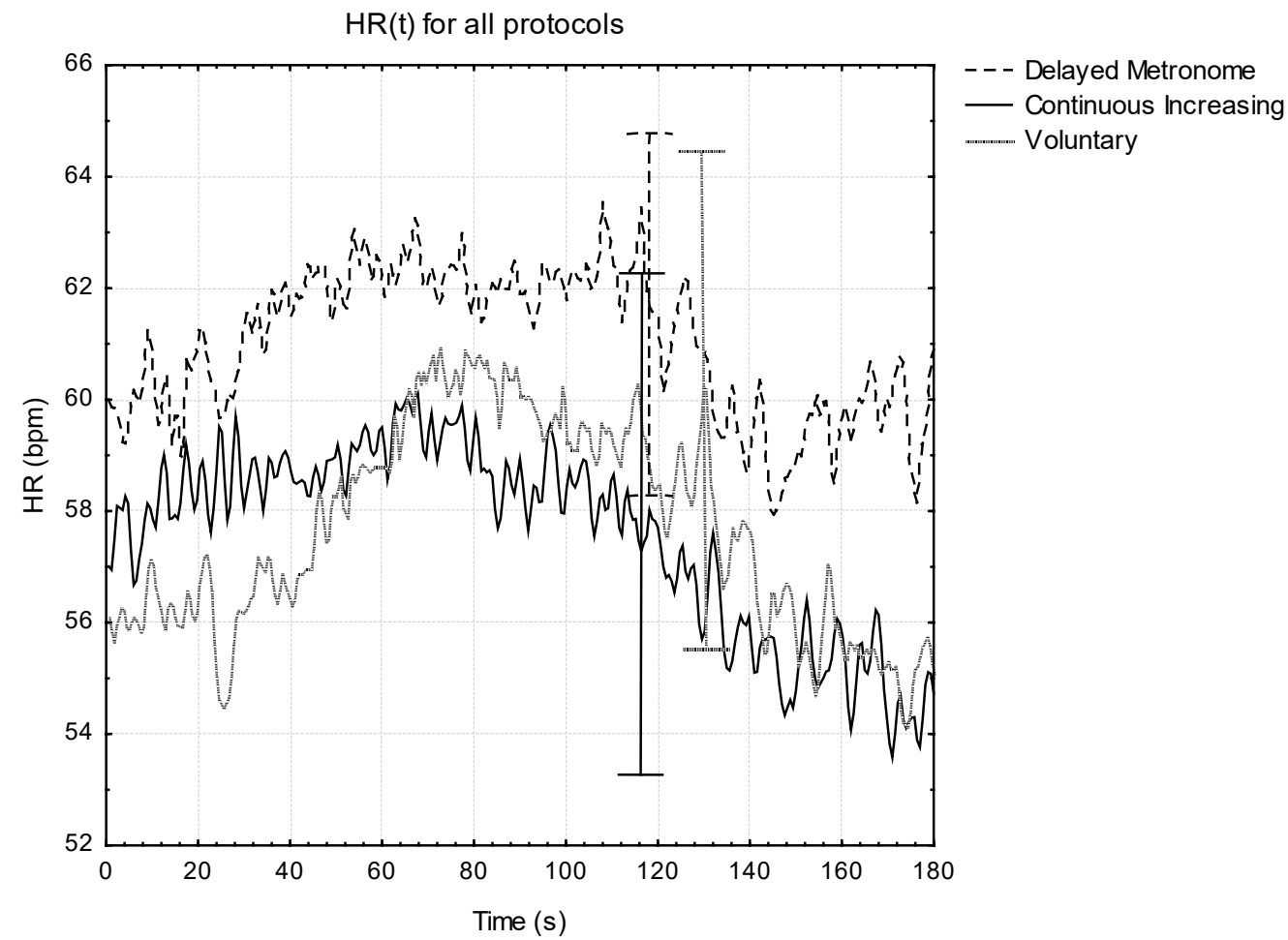


12 Figure 7: Coherent averages of $\mathrm{ABP}(\mathrm{t}), \mathrm{HR}(\mathrm{t}), \mathrm{CBFV}(\mathrm{t})$ and $\mathrm{EtCO}_{2}(\mathrm{t})$ for the maintenance protocol.

13 Shaded areas show error bars show \pm one standard deviation. Values of $p<0.05$ were considered 14 significant.
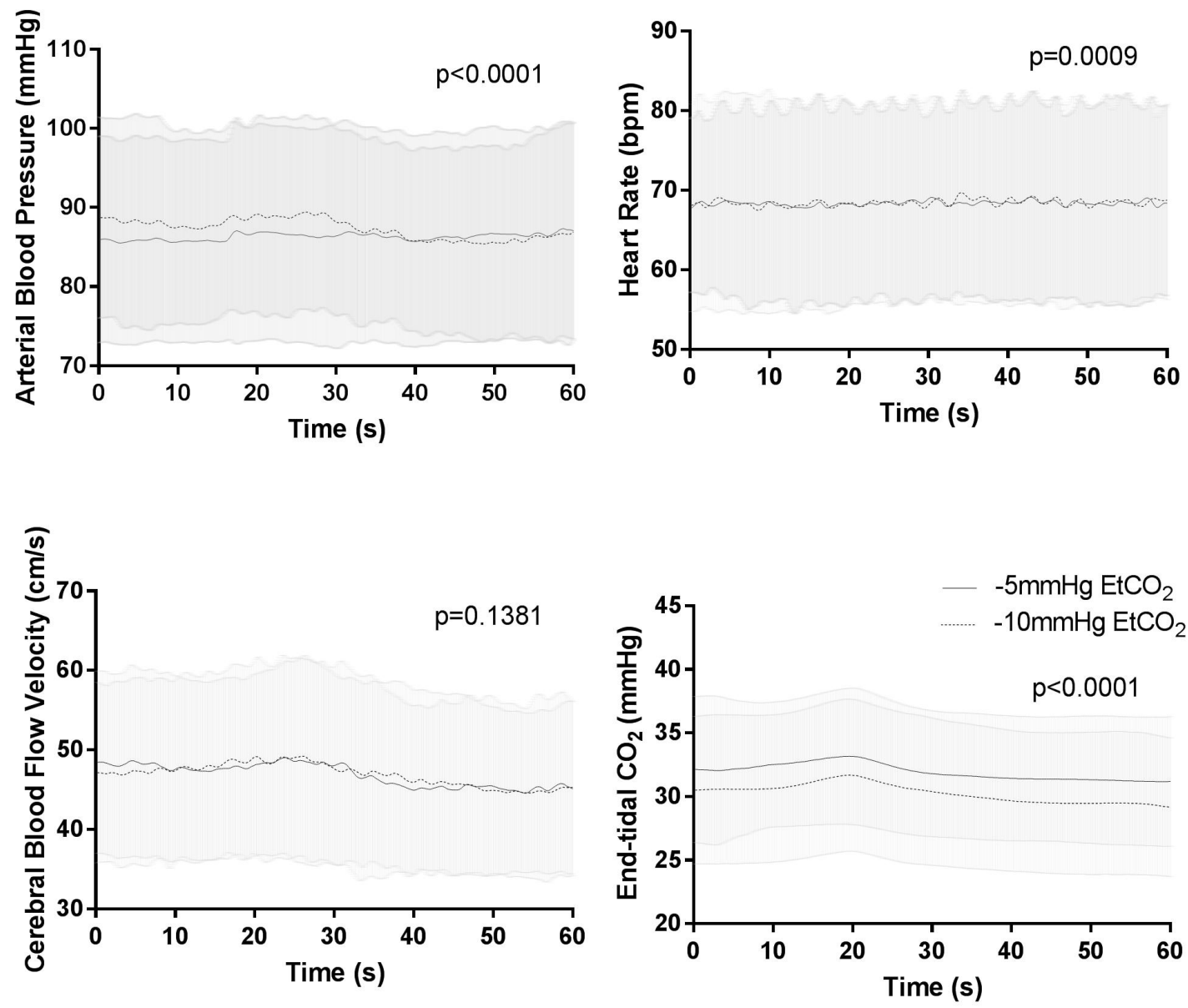